\title{
A Case of Metabolic Encephalopathy Triggered by Food Supplements
}

\author{
Nidhi Bansala, d, Divey Manocha ${ }^{\mathrm{b}}$, Liviu Danescu ${ }^{\mathrm{c}}$, Jennifer Kelly ${ }^{\mathrm{c}}$
}

\begin{abstract}
A 59-year-old Caucasian female with history of multiple sclerosis (MS), osteoporosis and breast cancer presented with weakness, constipation, confusion and short term memory loss. Her metabolic profile showed pre renal azotemia and severe hypercalcemia (16.2 $\mathrm{mg} \%$ ). Aggressive medical management led to favorable resolution of her symptoms. A complete diagnostic work up for the etiology of hypercalcemia was then performed. PTH levels were suppressed at $5.6 \mathrm{pg} / \mathrm{mL}$ arousing suspicion for hypercalcemia due to malignancy (prior history of breast cancer vs. new primary). PTHrP $(0.8$ $\mathrm{pmol} / \mathrm{L})$, TSH $(0.623 \mathrm{IU} / \mathrm{L})$ and free T4 $(2.87 \mathrm{ng} / \mathrm{dL})$ were within normal limits. 25- hydroxy Vitamin D level was high at $96 \mathrm{ng} /$ $\mathrm{mL}$, while 1-25 dihydroxy vitamin D was $28 \mathrm{pg} / \mathrm{mL}$. Review of her medication list showed daily intake of 800 units of vitamin D. The patient denied overdosing on prescription vitamin $\mathrm{D}$ but later admitted that she was ingesting an expensive naturally derived gel preparation containing about 16,000 IU of vitamin D3 daily. This was one of several alternative medications prescribed to her by an online practitioner for her MS and osteoporosis. Patient was counseled on potential side effects of excessive vitamin D supplementation and these were withheld at the time of discharge.
\end{abstract}

Keywords: Hypercalcemia; Vitamin D; Complementary and alternative medicine

\section{Introduction}

Complementary and alternative medications (CAM) re-

Manuscript accepted for publication January 31, 2013

${ }^{\text {a } D e p a r t m e n t ~ o f ~ M e d i c i n e, ~ S U N Y ~ U p s t a t e ~ M e d i c a l ~ U n i v e r s i t y, ~ N Y, ~}$ 13210, USA

${ }^{\mathrm{b}}$ Department of Gastroenterology, SUNY Upstate Medical University, NY, 13210, USA

${ }^{\mathrm{c}}$ Department of Endocrinology, SUNY Upstate Medical University, NY, 13210, USA

${ }^{\mathrm{d}}$ Corresponding author: Nidhi Bansal, 50 Presidential Plaza, Apt 908, Syracuse, NY, 13202, USA. Email: dr.nidi@gmail.com

doi: http://dx.doi.org/10.4021/jem146w ceive immense attention and utilization in today's era. The 2007 National Health Interview Survey (NHIS) showed that as many as 38 percent of American adults use CAM. Approximately 34 billion dollars yearly is spent on CAM in the U.S. alone. Popular amongst CAM are vitamin supplements, Chinese herbs, saw palmetto and fish oil. Individuals receive nutrients from dietary sources, but often take or are prescribed supplements such as fish oil and vitamin D by different health care providers including physicians, nurse practitioners, chiropractors and practitioners of alternative medicine. Due to a misconception on the part of the patient about the required dose and lack of communication between multiple providers, chronic ingestion of inappropriately high doses of such medications may take place resulting in life threatening complications [1]. We describe such a case of a patient with acute encephalopathy due to severe hypercalcemia associated with ingestion of inappropriately high doses of vitamin $\mathrm{D}$ supplements.

\section{Case Report}

A 59-year-old Caucasian female, financial consultant for a medicine practice with a past history of multiple sclerosis (MS), osteoporosis, breast cancer status post lumpectomy, radiation and chemotherapy was admitted with complaints of confusion, short term memory loss, headache, generalized weakness, and palpitations. Over the previous two weeks, she was found to be progressively confused, irritable, unable to walk and became wheelchair bound. She denied any weight loss, shortness of breath, chest pain, abdominal pain, fever or night sweats.

Her right-sided weakness secondary to MS were treated in the past with oral steroids, interferon Beta 1a and glatiramate acetate with partial relief of symptoms. She stated that she had not used MS medications for the past 10 years and her last flare-up was in 2001 when she responded well to a course of oral steroids. She denied drug allergies, smoking, illicit drug use or alcohol.

On physical examination, patient was confused, irritable; cranial nerve functions II - XII were grossly intact. Motor examination showed increased tone in bilateral lower 
extremities with $4 / 5$ strength and hyper reflexia in all four extremities. Sensory examination showed intact temperature and light touch with decreased vibratory sense in bilateral lower extremities. Plantar reflexes were upgoing bilaterally. Cardiovascular and rest of systemic examination was within normal limits.

Her metabolic profile showed pre-renal azotemia and severe hypercalcemia $(16.2 \mathrm{mg} \%)$. Initial management consisted of intravenous fluid bolus followed by a continuous drip titrated to a goal urine output of $100 \mathrm{~mL} /$ hour. Calcium levels slowly came down to a level of $7.9 \mathrm{mg} / \mathrm{dL}$. Her acute kidney injury also resolved and was likely due to hypercalcemia and dehydration. A complete diagnostic work up for hypercalcemia was then performed. PTH levels were suppressed at $5.6 \mathrm{pg} / \mathrm{mL}$ arousing suspicion for hypercalcemia due to malignancy (prior history of breast cancer vs new primary). However her PTHrP level was not elevated (0.8 $\mathrm{pmol} / \mathrm{L})$. TSH $(0.623 \mathrm{IU} / \mathrm{L})$ and free T4 $(2.87 \mathrm{ng} / \mathrm{dL})$ were also in normal range. Serum alkaline phosphatase levels (106 $\mathrm{IU} / \mathrm{L}$ ) were also normal 25 Hydroxy vitamin $\mathrm{D}$ was high at $96 \mathrm{ng} / \mathrm{mL}(10-55 \mathrm{ng} / \mathrm{mL})$, while 1,25 - Dihydroxy vitamin $\mathrm{D}$ was low normal at $28 \mathrm{pg} / \mathrm{mL}(10-65 \mathrm{pg} / \mathrm{mL})$.

Review of her medication showed that she was taking bupropion $300 \mathrm{mg}$ daily, tolterodine $4 \mathrm{mg}$ daily, diazepam 2 $\mathrm{mg}$ three times a day, gabapentin $600 \mathrm{mg}$ three times a day, pantoprazole $40 \mathrm{mg}$ daily, 800 units of vitamin D and 500 $\mathrm{mg}$ of calcium daily.

With limited sun exposure in Central New York weather, how our patient managed to achieve such a degree of symptomatic hypervitaminosis D was unclear. She denied overdosing on prescription vitamin D but later admitted to ingesting a naturally derived gel preparation containing approximately 16,000 international units of vitamin $\mathrm{D}$ daily for past 10 months. This was one of several medications she was prescribed online by a practitioner of alternative medicine to cure her multiple sclerosis and osteoporosis.

We counseled and provided information to the patient on the potential side effects of excessive vitamin D supplementation and other alternative preparations. She was instructed to discontinue all her supplements and was discharged from the hospital after complete recovery.

\section{Discussion}

Epidemiologically vitamin D supplementation has shown benefits in conditions such as osteoarthritis, multiple sclerosis, hypertension, insulin resistance, lipid abnormalities, mood affects and even prevention of some cancers $[2,3]$. The amount up to which a substance is tolerated without adverse effects in the majority of population is considered as its safe upper limit. Per the latest recommendations of the Institute of Medicine, the tolerable upper limit in adults for vitamin $\mathrm{D}$ is 4,000 IU/day [4]. The incremental consump- tion of $1 \mu \mathrm{g} / \mathrm{d}$ of vitamin D3 raises serum 25-hydroxyvitamin D (25(OH)D) by approximately $1 \mathrm{nmol} / \mathrm{L}(0.4 \mu \mathrm{g} / \mathrm{L})$. Published literature suggests that toxicity may occur with $25(\mathrm{OH}) \mathrm{D}$ concentrations beyond $500 \mathrm{nmol} / \mathrm{L}(200 \mu \mathrm{g} / \mathrm{L})$ [2]. Vitamin D toxicity may present clinically with nausea, vomiting, abdominal cramps, constipation, poor appetite, weight loss, confusion, perioral tingling sensations, weakness and heart rhythm abnormalities. Vitamin D is a fat soluble vitamin with a long half-life approximately 2 months, $25(\mathrm{OH}) \mathrm{D}$ which is the main transport form, has a half-life of approximately 15 days whereas the active 1, 25 dihydroxy vitamin $\mathrm{D}\left(1,25(\mathrm{OH})_{2} \mathrm{D}\right)$ has a half-life of approximately 15 hours [5].

In vitamin $\mathrm{D}$ toxicity due to excessive ingestion of cholecalciferol (25-hydroxyvitamin D3) or ergocalciferol (25-hydroxyvitamin D2), high levels of serum 25(OH)D with normal serum $1,25(\mathrm{OH})_{2} \mathrm{D}$ levels have been reported $[6,7]$. The liver converts almost all vitamin D to $25(\mathrm{OH})$ $\mathrm{D}$ through substrate-dependent cytochrome P450-linked oxidases which are loosely regulated [8]. Vitamin 25(OH)D therefore reflects the amount of parent vitamin D. It is likely that in cases of vitamin D toxicity, it is the $25(\mathrm{OH}) \mathrm{D}$ that is responsible for the hypercalcemia. At higher concentrations, vitamin D metabolites such as $25(\mathrm{OH}) \mathrm{D}$ may compete for binding at vitamin $\mathrm{D}$ receptor sites, thereby producing effects similar to those of $1,25(\mathrm{OH})_{2} \mathrm{D}$ by initiating translation of vitamin $\mathrm{D}$ receptor-responsive genes [9]. Also, higher concentrations of $25(\mathrm{OH}) \mathrm{D}$ may exceed vitamin $\mathrm{D}$ binding protein (DBP) binding capacity and free $25(\mathrm{OH}) \mathrm{D}$ enter the cells directly affecting the gene expression $[6,7,10]$.

The U.S. Food and Drug Administration (FDA) regulations for dietary supplements are not the same as those for prescription or over-the-counter drugs. In general, the regulations for dietary supplements are less strict and generally the manufacturer does not have to prove the safety and effectiveness of a dietary supplement before it is marketed. Once a dietary supplement is on the market, the FDA monitors safety and product information (label claims and package inserts), and the Federal Trade Commission (FTC) monitors advertising. The National Center for Complementary and Alternative Medicine (NCCAM) defines complementary and alternative medicine (CAM) as a group of diverse medical and health care systems, practices, and products that are not generally considered part of conventional medicine. It includes natural substances such as dietary supplements, probiotics, herbs; mind and body medicine like meditation, acupuncture, massage, reiki; behavioral therapy such as hypnotherapy, etc. Rigorous, well-designed clinical trials for many CAM therapies are often lacking; therefore, the safety and effectiveness of many CAM therapies are uncertain. Some dietary supplements may interact with medications or other supplements, may have side effects of their own, or may contain potentially harmful ingredients not listed on labels. Also most supplements have not been tested in pregnant 
women, nursing mothers, or children. Patients should notify all their health care providers about any complementary and alternative practices they are using so that they benefit from safe and coordinated care [11].

Patients should be reminded that "natural" does not always mean "safe". It is important to continue to monitor dietary supplements (not just herbal products) that may have interaction and toxicity issues. It is also equally important to educate patients and clinicians on supplements that do not have proven safety issues due to lack of robust studies, but have been shown in small laboratory studies and case reports to have safety concerns. There is a need to be vigilant and constantly updated about known and possible adverse effects. Medical providers should be aware of a patient's home environment and care team that may include family members and CAM practitioners recommending treatments outside of current established practice. These treatments in select cases may be deleterious to a patient's health and early detection by a traditional medical provider is likely the key to preventing possible adverse outcomes.

\section{Declaration}

Authors declare no conflicts of interest. Authors declare that no financial assistance was taken from any source. Authors declare that they have no commercial or proprietary interest in any drug, device, or equipment mentioned in the submitted article.

\section{References}

1. Moyad MA. Under-hyped and over-hyped drug-dietary supplement interactions and issues. Urol Nurs.
2010;30(1):85-87.

2. Vieth R. Vitamin D supplementation, 25-hydroxyvitamin D concentrations, and safety. Am J Clin Nutr. 1999;69(5):842-856.

3. Querfeld U, Mak RH. Vitamin D deficiency and toxicity in chronic kidney disease: in search of the therapeutic window. Pediatr Nephrol. 2010;25(12):2413-2430.

4. Institute Of Medicine (IOM) Dietary Reference Intakes for Calcium and Vitamin D. Available at: www.iom.edu. Accessed 20th August 2012.

5. Jones G. Pharmacokinetics of vitamin D toxicity. Am J Clin Nutr. 2008;88(2):582S-586S.

6. Jones G. Expanding role for vitamin D in chronic kidney disease: importance of blood 25-OH-D levels and extrarenal 1alpha-hydroxylase in the classical and nonclassical actions of 1alpha,25-dihydroxyvitamin D(3). Semin Dial. 2007;20(4):316-324.

7. Holick MF. Vitamin D deficiency. N Engl J Med. 2007;357(3):266-281.

8. G. Bilezikian JP, Raisz LG, Rodan GA, eds. Vitamin D and analogues. In: Jones Principles of bone biology. 3rd ed. San Diego, CA: Academic Press, 2008.

9. Prosser DE, Jones G. Enzymes involved in the activation and inactivation of vitamin D. Trends Biochem Sci. 2004;29(12):664-673.

10. Masuda S, Byford V, Arabian A, Sakai Y, Demay MB, St-Arnaud R, Jones G. Altered pharmacokinetics of 1alpha,25-dihydroxyvitamin D3 and 25-hydroxyvitamin D3 in the blood and tissues of the 25-hydroxyvitamin D24-hydroxylase (Cyp24a1) null mouse. Endocrinology. 2005;146(2):825-834.

11. National Center for Complementary and Alternative Medicine (NCCAM). Available at: http://nccam.nih. gov/health/whatiscam. Pub No:D347. Accessed 20th August 2012. 